\title{
A Parallel between Countability and Atomicity
}

\author{
Eun-joo Kwak \\ Sejong University
}

\begin{abstract}
The general notion of countability is that count nouns are pluralized and denote atomic entities while mass nouns are used only in singular forms and denote non-atomic entities. However, diverse linguistic data show that the correlation between countability and plurality is not tenable. In this study, challenges to the classical view are reviewed. Pointing out problems with the challenges, I argue that countability and atomicity are correlated.
\end{abstract}

Keywords: countability, atomicity, plurality, count nouns, mass nouns

\section{Introduction}

Traditionally, count nouns are pluralized and denote atomic entities while mass nouns are not used with plural forms and denote nonatomic entities. In other words, the three different notions of countability, plurality, and atomicity are parallel in this view. However, this close relation of the notions can be challenged in two respects. One is to reject the relation between countability and plurality, and the other 
is to argue against the relation between countability and atomicity. Some semanticists argue for the non-atomicity of count nouns and others for the atomicity of mass nouns.

To maintain the morpho-syntactic relations between countability and plurality, Chierchia (1998a, b) proposes that mass nouns are semantically plural, denoting atomic entities. Along with Chierchia, Rothstein (2008) accepts the plurality of mass nouns and argues for the necessity of contextually defined atoms for count nouns.

In this study, I focus on the interpretation domain of count and mass nouns. I argue that the semantic notions of countability and atomicity are interrelated and that count nouns must be interpreted in an atomic domain while the denotations of mass nouns are generated from non-atomic entities. I also discuss the diverse relations of countability and plurality cross-linguistically, showing that the two notions cannot be equated as in English.

\section{A Classical View}

\subsection{The Distinction between Count and Mass Nouns}

One way to define count and mass nouns is based on their morphosyntactic features. Since Jespersen (1909) first discussed the count/mass distinction, a major criterion for classifying the two categories is the availability of plural forms. Count nouns may occur in either singular or plural forms while mass nouns have only singular or numberneutral forms. This means that two different notions of countability and plurality do not have any discrepancy in their application. This paralleled relation leads to other linguistic phenomena. Cardinal numerals may modify only count nouns (e.g., one boy, two boys), but not mass nouns (e.g., *one water, *two furniture). Determiners are also divided into three classes: determiners for count nouns only (e.g., many, few) or mass nouns only (e.g., much, little), and determiners for both classes 
(e.g., some, most). Finally, since mass nouns may not be counted by themselves, they need measure phrases for counting as exemplified by a cup of water and a litre of water.

Another way to classify count and mass nouns is to consider their semantic properties. An argument on this topic by Quine (1960) states that if the meaning of a mass noun applies to each of two entities, it also applies to the two entities taken together.

(1) $a$. If $a$ is water and $b$ is water, then $a$ and $b$ together are water.

b. \#If $a$ is an apple and b is an apple, then $a$ and $b$ together are an apple.

When the property of being water is true for two separate entities $a$ and $b$, it also applies to the cumulative entity $a$ and $b$. This relation does not hold for a singular count noun like an apple as shown by the awkwardness of (1b). The distinct property of mass nouns in (1) is called 'cumulative reference' or 'cumulativity.' This is formally defined in (2), based on Krifka (1998).

(2) cumulativity

$\mathrm{P}$ is cumulative iff:

$\forall \mathrm{x} \forall \mathrm{y}[\mathrm{x} \in \mathrm{P} \wedge \mathrm{y} \in \mathrm{P} \wedge \mathrm{x} \neq \mathrm{y} \rightarrow \mathrm{x}+\mathrm{y} \in \mathrm{P}]$

$\mathrm{P}$ is a cumulative predicate iff for all $\mathrm{x}$ and $\mathrm{y}$ if $\mathrm{x}$ and $\mathrm{y}$ are in $\mathrm{P}$ and distinct, the sum of $\mathrm{x}$ and $\mathrm{y}$ is also in $\mathrm{P}$.

Another semantic property for mass nouns has been pointed out by Bunt (1985). The denotations of mass nouns behave as if they do not consist of discrete parts. In other words, mass nouns seem to consist of homogeneous masses. This means that if the meaning of a mass noun applies to an entity, then it applies to any smaller entity divided from the entity. ${ }^{1}$

${ }^{1}$ Quine (1960) rejects the divisivity of masses in that there may be parts of 
(3) a. Any part of something which is water is water.

b. \#Any part of something which is an apple is an apple.

(3a) shows that when some entity has the property of being water, all the smaller parts that are divided from the entity have the same property. This is called 'homogeneity' or 'divisivity.' Unlike the mass noun, the singular count noun an apple does not have a homogeneous denotation. Any parts that are divided from an apple cannot maintain the property of an apple, which is true only for the whole object. The property of homogeneity is assigned a formal definition as in (4).

(4) homogeneity

$\mathrm{P}$ is homogeneous iff:

$\forall \mathrm{x} \forall \mathrm{y}[\mathrm{x} \in \mathrm{P} \wedge \mathrm{y} \leq \mathrm{x} \wedge \mathrm{y} \neq \mathrm{x} \rightarrow \mathrm{y} \in \mathrm{P}]$

$\mathrm{P}$ is a homogeneous predicate iff for all $\mathrm{x}$ and $\mathrm{y}$ if $\mathrm{x}$ is in $\mathrm{P}$ and $\mathrm{y}$ is a proper part of $\mathrm{x}, \mathrm{y}$ is also in $\mathrm{P}$.

\subsection{An Interpretation Domain for Count and Mass Nouns}

Any interpretation for an expression is based on an interpretation domain, a model with all the entities that can be denoted. As discussed in the previous section, count and mass nouns are differentiated in

materials that are too small to retain the properties of larger entities. For example, when a drop of water is divided into the elements of hydrogen and oxygen, then these elements are not considered water any longer. To deal with this unwelcomed result, diverse solutions are suggested. For example, Bunt (1979) opts for the Minimal Parts Hypothesis, which argues for the postulation of minimal parts that are to be considered as materials. Link (1983), on the other hand, assumes that the material part-of relation of a lattice structure holds only in a mass domain. The restriction of domain for the material part-of relation has the effect of blocking awkward divisivity of water into hydrogen and oxygen. Another position for this problem is that the grammar is mute on the issue of minimal parts because a mass term like furniture seems to have minimal parts. (cf. Bunt 1985 and Gillon 1992) 
cumulativity and homogeneity. Hence, entities for count nouns need to be distinct from those for mass nouns to reflect these properties. Basically, an interpretation domain is assumed to consist of two different sets of entities: atomic and non-atomic entities. Atomic entities for count nouns are discrete. Discrete entities are identified by explicit boundaries in space that are separated from others, and they cannot be divided into smaller ones. The non-divisibility of atoms is also related to other properties: countability and finiteness. Atoms can be counted, and the number of atoms may be specified in a given structure. Non-atomic entities are for the denotations of mass nouns. Non-atomic entities are dense in that they may be infinitely divided into smaller entities. The density of non-atomic entities is connected with non-countability and infiniteness. For example, any amount of water can be divided into smaller portions of water, so we cannot say specifically how many entities of water are contained in a bottle of water.

Although count and mass nouns denote different entities, i.e., atoms and non-atoms, plural counts and masses share cumulativity. Singular count nouns are not subject to cumulativity. However, plural count nouns are cumulative like mass nouns. According to Quine, if the property of being horses applies to the animals of one camp and to those of another camp, the property also applies to the animals in the two camps. In other words, the property of being horses applies to the cumulated reference of the animals in the two camps.

Based on this similarity, Link (1983) proposes a 'complete join semi-lattice' for the structure of an interpretation domain. A join semilattice structure is defined by a join operation ' + ' and an individual part-of relation ' $\leq_{\mathrm{i}}$.' In this new structure, the plural term John and Mary refers to a 'sum' individual ' $\mathrm{j}+\mathrm{m}$,' a larger entity that is derived from the joining operation of $\mathrm{j}$ and $\mathrm{m}$. Furthermore, an individual part-of relation exists between this newly generated sum individual $j+m$ and the atomic individuals of $j$ and $m: j \leq j+m$ and $m$ $\leq_{i} j+m$. Here is a join semi-lattice for three individuals $j, m$, and $b$. 
(5) a. A Lattice for Count Nouns

b. A Lattice for Mass Nouns
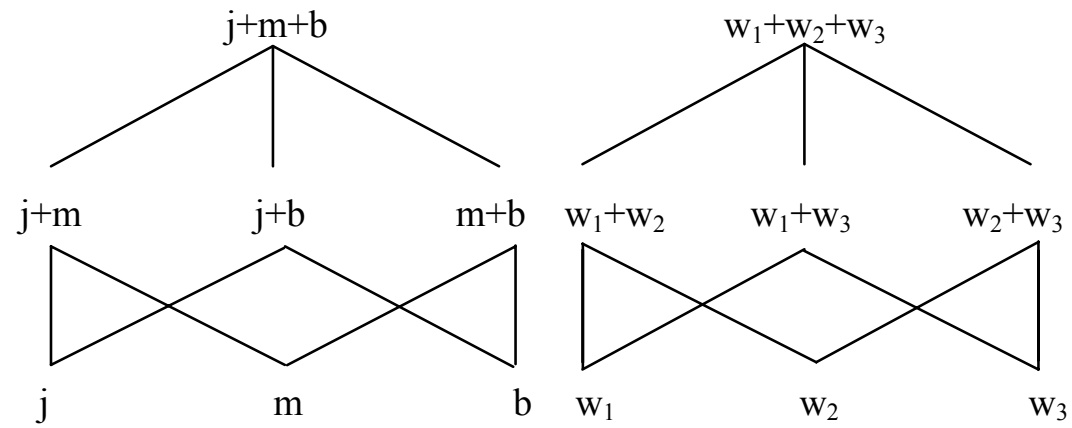

In the first tier in (5a), the atomic individuals, $\mathrm{j}, \mathrm{m}$, and $\mathrm{b}$, are located. Two of these individuals may be joined together to make a sum individual such as $\mathrm{j}+\mathrm{m}, \mathrm{j}+\mathrm{b}$, and $\mathrm{m}+\mathrm{b}$. In the third tier, all three individuals are joined to make the maximal sum $j+m+b$. Along with the lattice structure for plurals, Link also postulated another lattice structure for materials for mass nouns, which is defined by the join operation and a material part-of relation ' $\leq_{\mathrm{m}}$.' A cup of water $\mathrm{w}_{1}$ and another cup of water $\mathrm{w}_{2}$ may be poured into a basket to make a join material $\mathrm{w}_{1}+\mathrm{w}_{2}$. As with plurals, a material part-of relation holds between the sum material $\mathrm{w}_{1}+\mathrm{w}_{2}$ and their subparts $\mathrm{w}_{1}$ and $\mathrm{w}_{2}: \mathrm{w}_{1} \leq_{\mathrm{m}}$ $\mathrm{w}_{1}+\mathrm{w}_{2}$ and $\mathrm{w}_{2} \leq_{\mathrm{m}} \mathrm{w}_{1}+\mathrm{w}_{2}$. A join semi-lattice for three materials $\mathrm{w}_{1}$, $\mathrm{w}_{2}$, and $\mathrm{w}_{3}$ is represented in $(5 \mathrm{~b})$. It has the same structure as the lattice for individuals. Notice that although the two lattices for count and mass nouns are structured in the same way as in (5), they are generated from different entities. A lattice for count nouns is based on atomic entities while mass nouns have interpretations in a nonatomic lattice. Two different lattices generated from atoms and nonatoms properly reflect the common property between plurals and masses as well as the differences between singulars and masses.

Given the two separate lattices for count and mass nouns, Link (1984) and Landman (1989) argue that higher entities of 'groups' are needed in the domain to deal with the interpretations of collection terms 
like committee. Collection terms have ambivalent properties in that they are singular but have plural members internally. They may be modified by plural members as in a committee of women but not by singulars as shown by the awkwardness of a committee of woman. Hence, collection terms are regarded as denoting atoms because they can be pluralized as in committees, but they are generated from plural members. To deal with the dual nature of collection terms, Link and Landman propose that the lattice structures should be expanded to include higher entities of groups. Ordinary atoms are called 'pure' atoms, and groups, which are atoms generated from a sum of members, are called 'impure' atoms. To assign interrelations between pure and impure atoms, Link (1984) introduces a group formation function $\uparrow$, which maps a sum to a group. In addition, Landman (1989) expands on the structure with a member specification function $\downarrow$, mapping a group to a sum of its members.

The following example shows an expanded structure. Suppose that a police force consists of three officers, $j, m$, and $b$, and that the same group of people also take a class. In this situation, three individuals, $\mathrm{j}, \mathrm{m}$, and $\mathrm{b}$, the groups of the police force, and the class are all assumed to be atoms in the domain of (6).

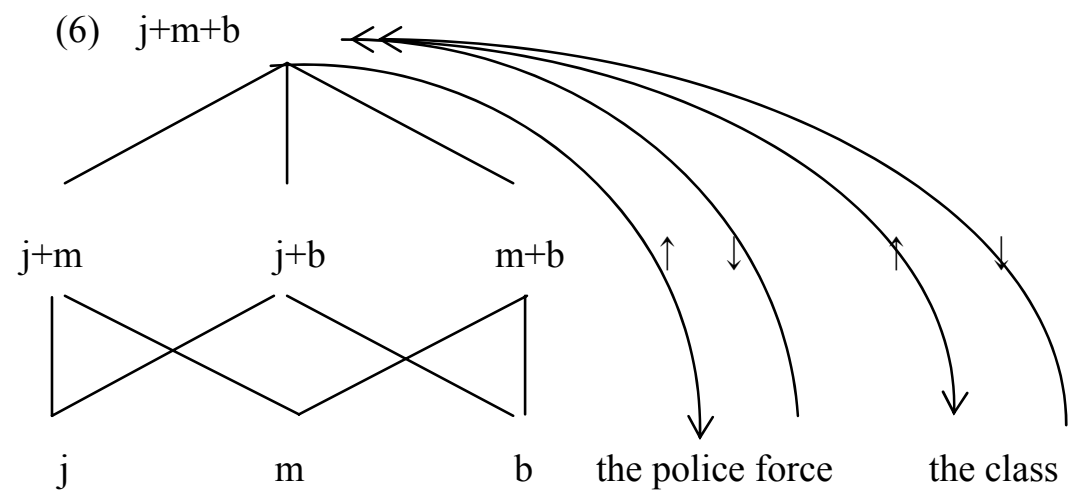


The sum $j+m+b$ is mapped to the police force or the class with $\uparrow .^{2}$ Likewise, the groups of the police force and the class are mapped to their members $\mathrm{j}+\mathrm{m}+\mathrm{b}$ with $\downarrow$. In this new structure, the police force denotes an atom like an ordinary NP such as John. However, it has an internal plural structure with its members, which is specified by $\downarrow$.

\section{Challenges to the Classical View}

The parallel relation between countability and atomicity varies according to different theories. An extensive argument against the parallel has been proposed by Fox \& Hackl (2006), in which count nouns as well as mass nouns denote non-atomic dense entities. They argue that the general density of the interpretation domain makes it possible to provide a better account for diverse linguistic phenomena related to measurement. It is debatable whether the postulation of density for count nouns has theoretical advantages. (cf. Kwak 2008) Even if their argument is correct, it mainly starts from the efficiency of explanations for some specified set of linguistic structures. It does not deal with the semantic properties of entities for count and mass nouns themselves. Hence, the proposal for non-atomic entities for count nouns is not covered in this study. Chierchia (1998a, b) and Rothstein (2008) display more serious arguments against the classical view. Chierchia provides typological study based on crosslinguistic data, arguing for the atomicity of mass nouns. Rothstein extends his analysis, focusing on the contextually affected atomicity of count nouns.

\footnotetext{
2 A sum of individuals $\mathrm{j}+\mathrm{m}+\mathrm{b}$ is mapped to two groups the police force and the class in (6), which is against the notion of function. Landman (1989) argues that the problem of mapping one to many is due to the intentional property of group. The same sum of individuals may act as different groups depending on a given situation. If we reinterpret the extensional structure of (6) in the intentional setting, this mapping problem does not occur.
} 


\subsection{The Atomicity of Mass Nouns}

Traditionally, arguments on mass nouns focus on the semantics of substance expressions like water and mud. Substances do not have minimal parts in the real world, and thus the assumption of non-atomic entities for mass nouns claims to model the world properly. For instance, a cup of water is infinitely divided into portions of water due to its homogeneity, so water is construed to denote nonatomic entities. ${ }^{3}$ In contrast with substances, mass collection terms such as furniture and silverware seem to have minimal parts. Furniture denotes a set of objects like tables and chairs, and the denotation of silverware consists of forks, spoons, and knives. Moreover, parts of their members like legs of tables or handles of spoons are not considered part of the denotations of collections. This goes against the homogeneity of mass nouns, thereby providing a basis for casting doubt on the atomic nature of mass nouns.

Unlike earlier theories, Chierchia (1998a, b) concentrates on the semantics of mass collections to delve into the semantics of mass nouns in general. He adopts the atomic nature of members for mass collections in the real world and argues for a model in which mass collections denote sets of atoms just like count nouns. Since furniture denotes entities that consist of pieces of furniture, Chierchia argues that there is no reason to introduce non-atomic entities other than atoms for pieces of furniture. Suppose that there are three pieces of furniture in a context: two chairs $c_{1}$ and $c_{2}$, and one table $t_{1}$. A singular term piece of furniture denotes a set of these three pieces as in (7a).

3 Although water is physically divided into atomic oxygen and hydrogen, this physical composition is not identical to the semantic structure of water. This is in the same line with the basic assumption that linguistic knowledge may be distinct from physical real world knowledge. 
(7) a. $[[$ piece of furniture $]]=\left\{\mathrm{c}_{1}, \mathrm{c}_{2}, \mathrm{t}_{1}\right\}$

b. $[[$ pieces of furniture $]]=\left\{\mathrm{c}_{1}, \mathrm{c}_{2}, \mathrm{t}_{1}, \mathrm{c}_{1}+\mathrm{c}_{2}, \mathrm{c}_{1}+\mathrm{t}_{1}, \mathrm{c}_{2}+\mathrm{t}_{1}, \mathrm{c}_{1}+\mathrm{c}_{2}+\mathrm{t}_{1}\right\}$

c. $[[$ furniture $]]=\left\{\mathrm{c}_{1}, \mathrm{c}_{2}, \mathrm{t}_{1}, \mathrm{c}_{1}+\mathrm{c}_{2}, \mathrm{c}_{1}+\mathrm{t}_{1}, \mathrm{c}_{2}+\mathrm{t}_{1}, \mathrm{c}_{1}+\mathrm{c}_{2}+\mathrm{t}_{1}\right\}$

Its plural counterpart pieces of furniture denotes a set closed under the sum operation in (7b). In Chierchia's analysis, the denotations of mass nouns are generated from atoms, and thus the mass noun furniture denotes a set of atoms closed under the sum operation in $(7 \mathrm{c})$. This amounts to the same set as the one in (7b), which means that the plural pieces of furniture and the mass furniture are interpreted in the same way.

Based on the equal denotations of plurals and mass collections, Chierchia argues that mass nouns are singular in their morpho-syntax but plural in their lexicon. Chierchia states that mass nouns come out of the lexicon with plurality already built in. He further argues that while the atoms of count nouns are grammatically accessible, those of mass nouns are not. To account for this distinction, the concept of atomicity is divided into two categories in his analysis. Formal atomicity, which addresses the atomic nature of an interpretation domain, is separated from semantic atomicity, i.e., atomicity visible in the semantics. Atoms in the denotations of count nouns are treated as atomic for both cases. However, atoms for mass nouns are atomic in an interpretation domain, but not treated as atomic in semantics. This is why Chierchia calls mass nouns lexically plural but grammatically singular.

Although Chierchia's arguments on mass collections seem to reflect the real world, mass nouns for substance readings are problematic with his analysis. If the object-like nature of collection members is the basis for proposing the atomicity of mass collections, the dense nature of substance like water is not accounted for in his analysis. In spite of this non-atomic nature of substances, Chierchia collapses collections and substances into one category of mass nouns, based on their identical morpho-syntactic properties. Included in the same 
category, mass nouns of substances are also assumed to denote atomic entities, which are the minimal relevant quantities of substances. What the minimal quantities are may be specified by context, or may be left vague and unspecified.

To provide a uniform treatment for collections and substances, Chierchia addresses their morpho-syntactic properties. First, mass nouns cannot be pluralized because they are already plural in the lexicon. Second, a term should denote a set of atoms to be counted. Since mass nouns denote sets of atoms closed under the sum operation, they cannot be directly counted like one furniture and two furniture. Third, the plurality of mass nouns requires additional classifier phrases or measure phrases to change their denotations into countable ones. This accounts for the distinction that count nouns do not occur with classifier or measure phrases but mass nouns do.

Chierchia's analysis diverges from the traditional view in several aspects. First, semantic atomicity is separated from formal atomicity. The atomic nature of entities does not guarantee that they are treated as atoms in semantics. Entities for mass nouns are atoms but not treated as atomic in semantics. Second, the distinction between count and mass nouns does not hinge on their atomicity but on their number properties. Expressions that may occur in plural forms are classified as count nouns, but expressions without plural forms are classified as mass nouns. According to this new criterion, languages may have only mass nouns when plural forms are not allowed in their grammar. For example, Chinese is assumed to have only mass nouns due to the lack of plural marking. Third, the structure of an interpretation domain may be affected by contexts. Although mass collections are consistently interpreted in an atomic domain, entities for substances cannot always be set to atoms. Hence, the interpretations of substances may vary depending on contexts. 


\subsection{Contextually Determined Atoms of Count Nouns}

Chierchia's (1998a, b) proposal for the atomicity of mass nouns is expanded by Rothstein (2008). Focusing on the semantics of count nouns, Rothstein argues against homogeneity as the common feature of mass nouns. Following Chierchia, Rothstein argues against the homogeneity of mass collections. Collections denote sets of objects but not their parts. For example, silverware denotes a set of forks, spoons, and knives, each of which denotes atomic entities. Moreover, parts of these objects like the handles of spoons are not included in the denotations of silverware, which does not follow the definition of homogeneity. In addition to the non-homogeneity of mass collections, Rothstein further argues that some substances have atomic parts in the real world. For instance, salt denotes entities that consist of salt grains, and a similar argument applies to rice. Since the smallest salt entities can be divided into entities that are not salt anymore, the substance salt is not fully homogeneous in its denotation. Note that the non-atomicity of mass nouns has been strongly supported by that fact that mass nouns denote homogeneous entities. Therefore, Rothstein concludes that the atomicity of mass nouns should be maintained.

Along with the partial homogeneity of mass nouns, Rothstein discusses the homogeneity of count nouns. According to Mittwoch (1988), the entities denoted by line may be divided into smaller entities which are lines themselves. In other words, a count noun line may have homogeneous parts. More examples of homogeneous count nouns are plane, sequence, twig, rope, fence, wall, hedge, and bouquet. (cf. Krifka 1992; Gillon 1992; Rothstein 2004, 2008) The existence of homogeneous parts means that the denotations of count nouns may not be generated from atoms in the old sense. To identify an atomic entity for line, there should be some operation to define a certain amount of string in a line.

As for the atomicity of homogeneous count nouns, Rothstein states that atomic interpretations may be affected by contexts. For 
example, if two adjoining houses have a fence between them and the street, the fence may be called either 'a fence' or 'two fences.' The singular 'a fence' is a better term when getting permission to build it, but the plural 'two fences' is more suitable when the owners ask for tax deductions for building them. Likewise, when the flowers of a bouquet are divided into two groups, each of them may be construed as a separate bouquet or just part of a bouquet. Therefore, Rothstein argues that an operation to define the atomicity of homogeneous count nouns should be contextually defined.

Based on the partial homogeneity of mass and count nouns, Rothstein proposes that an interpretation domain for nouns should be based on the semantics of root nouns, which provides a basis for the interpretations of count and mass nouns. Here is a summary of her proposal:

(8) An Interpretation Domain

a. Root nouns, $\mathrm{N}_{\text {root }}$, denote sets of minimal elements in $\mathrm{N}$ closed under plurality.

b. Mass nouns are root nouns: $\mathrm{N}_{\text {mass }}=\mathrm{N}_{\text {root }}$.

c. Count nouns, $\mathrm{N}_{\text {count }}$, denote sets of entities that measure 1 by the semantic operation M-ATOM: $\mathrm{N}_{\text {count }} \subseteq \mathrm{N}_{\text {root }}$.

Minimal elements are atoms for non-homogeneous count/mass nouns while they are minimal quantities for homogeneous count/mass nouns. As discussed by Chierchia (1998a, b), minimal quantities may be either specified by context or left vague. In other words, an interpretation domain may be indeterminate for minimal elements for some nouns. Given this, root nouns have denotations generated from atoms or minimal elements regardless of their countability. Once root nouns are assigned interpretations, the semantics of mass nouns is treated as identical to that of root nouns as stated in (8b). The interpretations of count nouns are rather complex because they need an additional semantic operation 'M-ATOM.' 
The operation of deriving $\mathrm{N}_{\text {count }}$ from $\mathrm{N}_{\text {root }}$ is called M-ATOM, which makes use of a standard measure function 'MEAS.' MEAS is a function from (singular and plural) individuals into ordered pairs where the first element is a natural number and the second element is a unit of measurement U. M-ATOM is a function from sets into sets which maps a set onto a subset of entities which count as one by a specified criterion.

(9) $\operatorname{M-ATOM}(\mathrm{N})=\lambda x[\mathrm{~N}(\mathrm{x}) \wedge \operatorname{MEAS}(\mathrm{x})=<1, \mathrm{U}>]$

The elements of M-ATOM(N) are the maximal elements that count as one N-entity in the context $\mathrm{U}$. They are called the M-ATOMs of $\mathrm{N}$ according to $\mathrm{U}$. The M-ATOM operation has the same effect of a maximalization operation, which gives the set of maximal nonoverlapping elements counting as one by a specified unit of measure. (cf. Filip \& Rothstein 2005)

Given the newly introduced M-ATOM, the denotations of count nouns are divided into two categories: contextually independent count nouns like boy and contextually defined ones like fence. Nouns that are naturally atomic are assigned interpretations with atoms the measurement of which follows the natural atomic structure of stuff. For example, the denotation of boy is a set of boys, whose atomicity is not affected by context.

$$
\begin{aligned}
& \text { (10) a. } {\left[\left[\mathrm{BOY}_{\text {count }}\right]\right]=\mathrm{M}-\operatorname{ATOM}\left(\left[\left[\mathrm{BOY}_{\text {root }}\right]\right]\right) } \\
&=\lambda \mathrm{x}[\mathrm{BOY}(\mathrm{x}) \wedge \operatorname{MEAS}(\mathrm{x})=<1, \mathrm{BOY}>] \\
& \text { b. }\left[\left[\mathrm{FENCE}_{\text {count }}\right]\right]=\mathrm{M}-\operatorname{ATOM}\left(\left[\left[\mathrm{FENCE}_{\text {root }}\right]\right]\right) \\
&=\lambda \mathrm{x}[\mathrm{FENCE}(\mathrm{x}) \wedge \operatorname{MEAS}(\mathrm{x})=<1, \mathrm{U}>]
\end{aligned}
$$

On the other hand, a homogeneous count noun like fence is not naturally atomic, and thus its entities do not come in inherently individuated units. Instead, it denotes a set of entities, the atomicity of which is determined by a context variable $U$. 
Rothstein defines an interpretation domain to be generated from the semantics of root nouns. The denotations of mass nouns are identical to those of root nouns while those of count nouns are derived by the application of M-ATOM to root nouns. No matter how count and mass nouns are assigned denotations, they are based on the minimal elements of root nouns. This means that there could be cases that count and mass nouns denote the same set of objects with no distinction. Following Chierchia, Rothstein argues that what really distinguishes count and mass nouns is their different grammatical accessibility. Even if the same minimal elements are assigned to a pair of a count and a mass, only those of the count are semantically atomic and thus grammatically accessible.

To show the different accessibility of count and mass nouns, Rothstein argues that the atoms of mass nouns are not visible in the distributivity of a reciprocal, following Gillon (1992). The minimal pair, curtains and curtaining, denotes the same objects but are different only in their countability. Carpets and carpeting represent another example of minimal pairs. When these minimal pairs occur in sentences, they show different patterns of distributivity.

(11) a. The curtains and the carpets resemble each other.

b. The curtaining and the carpeting resemble each other.

The conjunction of the count nouns in (11a) triggers an ambiguity between individual distributivity and group distributivity. It may be construed that the curtains resemble each other and the carpets resemble each other, or that the curtains as a whole resemble the carpets. However, the conjunction of the mass nouns in (11b) only induces distributivity between the two groups. Distributivity between individual curtains or carpets is not possible in (11b). The atoms of the count nouns are defined by M-ATOM, and thus the individual curtains or the group of the curtains are contextually identified as atoms. This is the source of the ambiguity in (11a). However, the 
atoms for the curtaining are not individually accessible, which leads to the group reading.

\section{Arguments for the Classical View}

\subsection{The Non-atomicity of Mass Nouns}

The atomicity of mass nouns is argued against by Chierchia (1998a, b) and Rothstein (2008). Chierchia aims for typological study with the cross-linguistic distribution of bare nouns in argument positions. To achieve this, he focuses on morpho-syntactic properties of nouns and argues that bare nouns without articles and number inflection are mass. He further proposes that mass nouns denote plural entities generated from atoms. Rothstein argues against the homogeneity of mass nouns. Since homogeneity works as strong evidence for the non-atomicity of mass nouns, partial non-homogeneity may be regarded as theory-external evidence for the atomicity of mass nouns.

Chierchia's argument for the atomicity of mass nouns hinges on their morpho-syntactic properties. According to Chierchia, bare nouns are mass and cannot take plural marking because they are already lexically plural. This typological prediction faces counter examples when analyzed cross-linguistically. Bare nouns are claimed to be optionally pluralized in diverse languages including Korean, Japanese, Indonesian, and Turkish. For instance, singular-formed count nouns in Korean show ambiguity between singular and plural readings while plural-formed count nouns are interpreted as plurals.
(12) a. namca
'a/the man or (the) men'
man
b. namca-tul
'(the) men'
man-PL 
Count nouns are pluralized whereas mass nouns are not in Chierchia's analysis. Given the optionality of the plural marker -tul, Korean count and mass nouns cannot be classified as Chierchia argues. The numberbased distinction is more problematic with the diverse patterns of number specification for mass nouns. According to Corbett (2000), mass nouns in Turkana and Bantu languages are divided between singulars and plurals while all mass nouns in Manam occur as plurals. Based on these observations, Nicolas (2008) argues that a grammatical number has no semantic import for mass nouns. Given the optional plurality of count nouns and diverse patterns of plurality for mass nouns, bare nouns cannot be equated with mass nouns.

Chierchia also argues that although mass nouns denote plural entities generated from atoms, these atoms are not visible in semantics. Hence, mass nouns need a classifier or measure phrase to be counted, but count nouns do not need this because their atoms are grammatically accessible. This claim is not tenable with Korean plurals. Namca-tul 'men' is a count noun since it is plural-marked.

(13) a. sey namca-tul

'three men' three man-PL

b. sey myeng-uy namca-tul 'three men' three CL-Poss man-PL

This count noun may be accompanied by a classifier phrase as in (13b). Then, the occurrence of a classifier phrase may not be used as semantic evidence for non-countability. This means that grammatical accessibility is not checked by the occurrence of a classifier phrase.

Since bare nouns are treated as mass in Chierchia's analysis, languages without plural marking in the grammar are assumed to have only mass nouns. However, Wilhelm (2008) argues that plural marking is separate from countability. Dëne Suliné (henceforth Dëne), a Northern Athapaskan language spoken in Northern Canada, is a bare noun language which lacks not only articles or number inflection 
for nouns but also numeral classifiers. Hence, Dëne bare nouns may combine directly with a numeral.
(14) solághe k'ásba
'five chickens'
five
chicken

A bare noun k'ásba 'chicken,' which should be classified as mass in Chierchia's analysis, does not need a classifier or measure phrase to be counted. Interestingly, not all bare nouns may combine directly with numerals, but nouns of liquid and substance readings need a measure or container phrase to be counted.
(15) a. \#solághe ejëretth'úé
'five milk'
b. \# solághe bër
'five meat'
(16) a. solághe ejëretth'úé tılı 'five pounds of milk' five milk container
b. solághe nedádhi bër 'five cartons of meat' five pound meat

Based on the fact that homogeneous nouns need a measure or container phrase, Wilhelm argues that while the count/mass distinction does exist in Dëne, plural marking has no role in this distinction.

Chierchia's morpho-syntactic arguments for the atomicity of mass nouns do not hold against many counterexamples cross-linguistically. However, to draw any conclusion on atomicity, one must review the semantic property of mass nouns. Rothstein as well as Chierchia, deny homogeneity as a common feature of mass nouns. This works as evidence for the atomic nature of mass entities. The count/mass distinction is independent of the structure of matter, and thus the homogeneity of mass nouns does not address whether materials for a given expression are physically homogeneous or not. ${ }^{4}$

${ }^{4}$ Chierchia (1998a, b) enumerates mismatched examples between real world 
To see whether mass nouns are homogeneous, let us compare count and mass collections. Collection terms denote entities that have plural members. Along with mass nouns like furniture and silverware, collection readings are also delivered by count ones like fleet and gaggle. Although both count and mass collection nouns have plural members, only count collections allow their members to be accessed in grammar. Here are examples from Nicolas (2008):

\section{(17) a. a fleet of ships, a gaggle of geese \\ b. \#furniture of tables, \#silverware of knives}

When plural members of ships constitute a collection fleet, they may be explicitly specified by modification as in (17a). Similarly, $a$ gaggle may be modified by its members. Modification by its members means that the denotation of a collection may be distinguished by the identities of its members. A fleet may be modified by ships only when it is semantically distinct from a fleet of fighters. Note that this modification is not allowed for the mass collections as in (17b). Furniture cannot be modified by tables even in the situation that it has only tables as its members. Members like tables and chairs cannot be used to specify the denotation of a mass collection. This means that members of a mass collection are treated as homogeneous without having independent identities in semantics.

The homogeneity of members is further shown by the fact that the properties of their members are not projected to mass collections. Although the denotations of coin and change are based on the same objects, they do not share all the properties. The plural coins may

atomicity and semantic atomicity. Entities that consist of natural units of equal perceptual salience may be expressed by count or mass nouns. For instance, rice is mass while lentil is count. Additionally, the same entities may be expressed by pairs of synonyms which are differentiated only in their countability. It is exemplified by the pairs of coins/change, shoes/footwear, carpets/carpeting, hair/hairs, and stone/stones. 
inherit the properties of its members, but the mass collection change may not. When a group of coins are divided into silver and gold ones, the distinct properties of its members are passed to the sum of coins.

(18) a. gold and silver coins

b. \#gold and silver change

However, the heterogeneous properties are not adopted for the denotation of the mass change. Only properties for a whole may be applied to the mass collection as shown by small change. The contrast shown in (18) is easily understood when the members of a mass collection are homogeneous regardless of their distinctness in the real world.

The arguments for the atomicity of mass nouns are defended by their morpho-syntactic patterns and the partial non-homogeneity of their denotations. However, as discussed in this section, languages show diverse patterns in the relations between countability and plurality, thereby weakening Chierhchia's typological analysis. Additionally, members of mass collections are treated as homogeneous in semantics despite their possible distinctness in the real world. Therefore, there is no reason to assume that mass nouns are interpreted in an atomic domain.

\subsection{An Interpretation Domain with Atoms and Non-atoms}

In the classical view, an interpretation domain is a join semilattice that consists of atoms for count nouns and non-atoms for mass nouns. To follow this structure, we need to deal with the dense nature of homogeneous count nouns like line and bouquet. As small parts of a line may be understood as a line, atoms for a line are hard to define in the current structure. Likewise, part of a bouquet may also be construed as a separate bouquet. The density of line is to determine how small lengths of line should be accepted as atoms. 
For bouquet, we must decide how many flowers are needed to make one bouquet. In Rothstein's (2008) analysis, the minimal quantities for line or bouquet, which may remain indeterminate without contexts, are specified with the semantic operation M-ATOM.

Before moving to the structure of a domain, the minimal part problem is not restricted to homogeneous count nouns. It is common with diverse entities such as events, time, space, and degrees. Moreover, the minimal quantity or size for these entities is affected by contexts. Here is an example from Landman, in which the same event is described in the contexts of different granularity levels.

(19) Yesterday afternoon between twelve and two I was sleeping; I woke up when you called me; in fact, at the moment you called me, I woke up; more precisely, at the moment that the telephone first rang, I woke up; in fact I woke up at the moment the telephone first started ringing; to be more precise, I woke up when the first sound of the first ring started, etc.

Wake up, an achievement predicate, is understood to denote an atomic event, and thus the time that you called me is considered as an atom in the temporal structure. However, this temporal atom is further divided into smaller ones in the following descriptions the moment you called me, the moment that the telephone first rang, etc. This shows that the size of an atom is affected by contexts. An atom in a coarse-grained structure may be mapped to a sum of entities in a more fine-grained structure.

To deal with multiple contexts defined by granularity levels, Link (1987) proposes a multi-layered structure of events that are sorted by granularity.

(20) $E$ is a system of lattices $\left(E_{i}\right)_{i \in J}$, where $J$ is a partially ordered set of indices which represent a certain granularity 
of the events in the corresponding lattice.

The event structure $E$ consists of multiple event structures $E_{l}, \ldots$ $E_{i}, \ldots$, which are different conceptualizations of the same realm of phenomena. These structures are partially ordered by granularity, as represented by the index. Here, the events in $E_{i}$ are more finegrained than those in $E_{j}$ for $\mathrm{i} \leq \mathrm{j}$. This multi-layered structure may apply to other categories of entities such as individuals, materials, times, and spaces because they show the same granularity problem as events.

In a multi-layered structure, the minimal quantity to define atoms is determined by the granularity level of a context. For example, some geometrical object may be understood as a point in a coarse-grained structure but as a line in a more fine-grained one. The set of atoms for line may be varied by the granularity level of context. However, once a granularity level is set, line denotes a set of atoms in a given structure. Hence, a join semi-lattice with a multi-layered structure has no indeterminacy. This means that the possible incompleteness of a domain described by Chierchia (1998a, b) and Rothstein (2008) is not relevant to the current structure.

The function of Rothstein's M-ATOM is actually divided into two: to define atoms and groups. It is about defining an (a?) (pure) atom to determine how small entities' atoms for line are, and it is about defining an impure atom of group to determine how many flowers are needed for a bouquet. Atoms and groups are collapsed in the operation of M-ATOM in Rothstein's analysis. However, in a multi-layered structure, the size of an atom is determined by the granularity level of context, and a group is defined only when the context has a strong necessity to make it a whole. One flower cannot make a bouquet by the collective definition of bouquet. Whether a bunch of flowers is accepted as a bouquet is affected by its composition. When the flowers make some structure to make them a whole, they can be understood as more than a sum of flowers and as a group 
bouquet. This is the ordinary concept of the group explained by Link (1984) and Landman (1989). Thus, no additional operation is needed for the current structure. Count and mass nouns are properly interpreted in a domain of a join semi-lattice sorted by granularity, which has atoms for count nouns and non-atoms for mass nouns.

\section{Conclusion}

The classical view on the distinction between count and mass nouns is that count nouns are pluralized while mass nouns are not. The correlation between countability and plurality further leads to a semantic relation in that count nouns denote atomic entities and mass nouns denote non-atomic entities. The morpho-syntactic correlation between countability and plurality does not seem to be maintained with cross-linguistic data which show diverse patterns. Hence, theories can be modified in two ways. One is to keep the morpho-syntactic correlation between countability and plurality while abandoning the semantic correlation between countability and atomicity. The other is to stick to the semantic correlation and sacrifice the morphosyntactic one. Chierchia (1998a, b) and Rothstein (2008) adhere to the former, while Link (1983) and Landman (1989) follow the latter.

I have shown that Chierchia and Rothstein's assumption that mass nouns are plural and denote atomic entities is not always correct. Morpho-syntactic evidence in diverse languages shows that plurality is a separate issue from countability. Hence, any semantic argument based on this correlation will fail. I have also argued that the homogeneity of mass nouns is tenable in spite of the apparent nonhomogeneity of mass nouns and partial homogeneity of count nouns. Since the homogeneity of mass nouns works as a theoretical ground for non-atomic entities of mass nouns, the semantic correlation between countability and atomicity is still correct. Finally, I have discussed the double function of M-ATOM by Rothstein and concluded that a 
136 A Parallel between Countability and Atomicity

join semi-lattice sorted by granularity is an appropriate structure for an interpretation domain.

\section{References}

Bunt, H. 1979. Ensembles and the Formal Semantic Properties of Mass Terms. In J. Pelletier (ed.), Mass Terms: Some Philosophical Problems 249-270. Dordrecht: Reidel.

. 1985. Mass Terms and Model-theoretic Semantics. Cambridge:

Cambridge University Press.

Cheng, L. \& R. Sybesma. 1999. Bare and Not-so-bare Nouns and the Structure of NP. Linguistic Inquiry 30, 509-542.

Chierchia, G. 1998a. Plurality of Mass Nouns and the Notion of Semantic Parameter. In S. Rothstein (ed.), Events and Grammar. Dordrecht: Kluwer.

. 1998b. Reference to Kinds Across Languages. Natural Language and Semantics 6, 339-405.

Corbett, G. 2000. Number. Cambridge: Cambridge University Press.

Doetjes, J. 1997. Quantifiers and Selection. On the Distribution of Quantifying Expressions in French, Dutch and English. Leiden: Holland Academic Graphics.

Filip, H. \& S. Rothstein. 2005. Telicity as a Semantic Parameter. In J. Lavine et al. (eds.), The Princeton University Meeting 139-156. Ann Arbor: Michigan Slavic Publications.

Fox, D. \& M. Hackl. 2006. The Universal Density of Measurement. Linguistics and Philosophy 29, 537-586.

Gillon, B. 1992. Toward a Common Semantics for English Count and Mass Nouns. Linguistics and Philosophy 15, 597-640.

Krifka, M. 1992. Thematic Relations as Links between Nominal Reference and Temporal Constitution. In I. Sag \& A. Szabolsci (eds.), Lexical Matters. Chicago, IL: The University of Chicago Press. 
Krifka, M. 1998. The Origins of Telicity. In S. Rothstein (ed.), Events and Grammar. Dordrecht: Kluwer.

Kwak, E. 2008. A Density Problem in Semantics. Korean Journal of Linguistics 33, 203-227.

Landman, F. 1989. Groups I \& II. Linguistics and Philosophy 12, 559-606 \& 723-744. . 1991. Structures for Semantics. Dordrecht: Kluwer. . 1983. The Logical Analysis of Plurals and Mass Terms: A Lattice Theoretical Approach. In N. Bäuerle et al. (eds.), Meaning, Use, and Interpretation of Language 302-323. Berlin: Walter de Gruyter.

. 1984. Hydreas on the Logic of Relative Construction with Multiple Heads. In F. Landman et al. (eds.), Varieties of Formal Semantics 245-257. Dordrecht: Foris.

. 1987. Algebraic Semantics of Event Structures. In J. Groenendijk et al. (eds.), Proceedings of the Sixth Amsterdam Colloquium. ITLI.

Mittwoch, A. 1988. Aspects of English Aspect; on the Interpretation of Perfect, Progressive and Durational Phrases. Linguistics and Philosophy 11, 203-254.

Nicolas, D. 2008. Mass Nouns and Plural Logic. Linguistic and Philosophy 31.2, 211-244.

Quine W. V. 1960. Word and Object. Cambridge: MIT Press.

Rothstein, S. 2004. Structuring Events: A Study in the Semantics of Lexical Aspect. Oxford: Blackwell. . 2007. Counting and the Mass/Count Distinction. Ms. Bar-Ilan University.

Wilhelm, A. 2008. Bare Nouns and Number in Dëne Suliné. Natural Language Semantics 16, 39-68. 